\title{
On the Outage Probability of MIMO Full-Duplex Relaying : Impact of Antenna Correlation and Imperfect CSI
}

DOI:

10.1109/TVT.2016.2602238

\section{Document Version}

Accepted author manuscript

Link to publication record in Manchester Research Explorer

\section{Citation for published version (APA):}

Almradi, A., \& Hamdi, K. (2016). On the Outage Probability of MIMO Full-Duplex Relaying : Impact of Antenna Correlation and Imperfect CSI. IEEE Transactions on Vehicular Technology, PP(99).

https://doi.org/10.1109/TVT.2016.2602238

\section{Published in:}

IEEE Transactions on Vehicular Technology

\section{Citing this paper}

Please note that where the full-text provided on Manchester Research Explorer is the Author Accepted Manuscript or Proof version this may differ from the final Published version. If citing, it is advised that you check and use the publisher's definitive version.

\section{General rights}

Copyright and moral rights for the publications made accessible in the Research Explorer are retained by the authors and/or other copyright owners and it is a condition of accessing publications that users recognise and abide by the legal requirements associated with these rights.

\section{Takedown policy}

If you believe that this document breaches copyright please refer to the University of Manchester's Takedown Procedures [http://man.ac.uk/04Y6Bo] or contact uml.scholarlycommunications@manchester.ac.uk providing relevant details, so we can investigate your claim.

\section{OPEN ACCESS}




\title{
On the Outage Probability of MIMO Full-Duplex Relaying : Impact of Antenna Correlation and Imperfect CSI
}

\author{
Ahmed Almradi, Student Member, IEEE, and Khairi Ashour Hamdi, Senior Member, IEEE
}

\begin{abstract}
This paper analyzes the performance of multipleinput multiple-output (MIMO) full-duplex (FD) relaying systems, where the source and destination nodes are equipped with single antenna and communicating via a dual-hop amplify-andforward (AF) relay with multiple receive and transmit antennas. The system performance due to practical wireless transmission impairments of spatial fading correlation and imperfect channel state information (CSI) is investigated. At the relay, the loopback self-interference $(\mathrm{LI})$ is mitigated by using receive zero-forcing (ZF) precoding scheme, then steering the signal to the destination by using maximum-ratio transmission (MRT) technique. To this end, new exact closed-form expressions for the outage probability are derived, where the case of arbitrary, exponential, and no correlations are considered. Meanwhile, for a better system performance insights, simpler outage probability lower-bound expressions are also included, through which the acheiveable diversity order of the receive $\mathrm{ZF} / \mathrm{MRT}$ scheme is shown to be $\min \left(N_{R}-1, N_{T}\right)$, where $N_{R}$ and $N_{T}$ are the number of relay receive and transmit antennas, respectively. Numerical results sustained by Monte Carlo simulations show the exactness and tightness of the proposed closed-form exact and lower-bound expressions, respectively. In addition, it is seen that the outage probability performance of FD relaying outperforms that of the conventional half-duplex (HD) relaying at low to medium signalto-noise ratio (SNR). However, at high SNR, the performance of HD relaying outperforms that of the FD relaying. Furthermore, in the presence of channel estimation errors, an outage probability error floor is seen at high SNR. Therefore, for optimum outage performance, hybrid relaying modes is proposed which switches between HD and FD relaying modes.
\end{abstract}

Index Terms-MIMO relaying, full-duplex relaying, halfduplex relaying, maximum-ratio combining (MRC), maximum ratio transmission (MRT), zero-forcing $(\mathrm{ZF})$, outage probability.

\section{INTRODUCTION}

$\mathbf{C}$ OOPERATIVE relaying techniques have gained a great deal of attention due to their ability to extend network coverage, connectivity and attain higher capacity without sacrificing extra power resources. In a dual-hop relaying systems,

Copyright (c) 2015 IEEE. Personal use of this material is permitted. However, permission to use this material for any other purposes must be obtained from the IEEE by sending a request to pubs-permissions@ieee.org.

A. Almradi is with the Department of Electrical and Electronic Engineering, Azzaytuna University, Tarhuna, Libya. He is also with the school of Electrical and Electronic Engineering, The University of Manchester, Manchester, M13 9PL, UK. (e-mail: elmaradi@gmail.com).

K. A. Hamdi is with the school of Electrical and Electronic Engineering, The University of Manchester, Manchester, M13 9PL, UK. (e-mail: k.hamdi@manchester.ac.uk).

Part of this paper was presented at the IEEE Wireless Communications and Networking Conference (WCNC), Doha, Qatar, April 2016. an intermediate idle node operates as a relay between the source and destination nodes when the direct link between the source and destination is in deep fade. Two orthogonal channels are required for communications to take place in the conventional dual-hop one-way relaying networks. As a result, a significant loss of spectrum efficincy is incurred because of the inherent half-duplex (HD) relaying transmission constraint at the relay (in the HD mode, time-division duplex or frequency-division duplex is utilized to assure orthogonal transmission (also known as out-of-band full-duplex)). Recently, full-duplex (FD) relaying, also known as in-band full-duplex, has received a lot of research interest because of its potential to double spectral efficiency (see e.g., [1][13]). This is due to the fact that full-duplex relays receive and re-transmit its information at the same time over the same frequency. Hence, efficiently utilizing the spectrum resources of the system. However, the main limitation of FD relaying is the loopback self-interference (LI) caused by the signal leakage from the relay's transmission to its own reception, namely, the large power differences between the LI (power transmitted from the FD relay) and the FD relay received signal (which is much weaker than the transmitted signal due to path loss and fading) exceeds the dynamic range of the analoge-to-digital converter. Therefore, LI mitigation and cancellation is vital in the implementation of FD relaying operation [1]-[6], [14].

The ever increasing demand of wireless communication devices with higher throughput led to the deployments of short range systems, such as small-cell systems, $\mathrm{WiFi}$, and Femtocells, where the cell-edge path loss is much less than that in the traditional cellular systems. Therefore, the transmission power and distance between devices has been decreased. This significant constructural modification alongside with the recent advances in antennas and radio-frequency (RF) circuit design is the breakthrough in making full duplex communications feasible as the LI mitigation problem becomes viable. Several LI mitigation techniques have been proposed in the literature which may be categorized into three stages: propagation (passive) domain LI cancellation, analog and digital (active) domain LI cancellation, spatial domain LI cancellation in the presence of multiple receive and/or multiple transmit antennas at the FD relay [3], [5], [6], [10]. Meanwhile, simultaneous reception and transmission in the FD mode requires the relay to have a separate transmit and receive RF chains. In addition, theoretically, FD systems can be equipped with either shared antenna or separate antennas. In shared antenna FD systems, 
only one antenna is used for both transmission and reception simultaneously, where a circulator is used to separate the received and transmitted signals. In separate antennas FD systems, two antennas are required, one for reception and the other for transmission, where natural isolation is used as a first step LI reduction between the transmit and receive antennas by using path loss. It is to be emphasized that in the case of multiple antennas FD systems, it is challenging to use shared antennas in multiple antennas FD systems as it results in sever interefernce between the shared antennas [3], [5].

Linking multiple-input-multiple-output (MIMO) with relaying systems substantially increase capacity and improve reliability. While most previously published work has focused on MIMO HD relaying transmission (see e.g., [15]-[18]), recent work has also considered the combination of MIMO techniques with FD relaying transmission mode. MIMO FD relaying transmission offer a powerful technique of suppressing the LI in the spatial domain and can offer higher capacity than the conventional MIMO HD relaying mode (e.g., [10], [11]). Therefore, in the presence of MIMO FD relaying systems, joint optimization by precoding and decoding at the transmitter and receiver, respectively, can be used to mitigate the LI effects. Zero-forcing (ZF) precoding and decoding vectors based on the conventional singular value decomposition (SVD) of the LI channel is proposed in [10] to null out the LI at the relay. In addition, for simplicity and mathematical tractability, a low complexity joint precoding/decoding design for maximizing the overall signal-to-noise ratio (SNR) is investigated in [11], where a closed-form overall SNR is derived. More specifically, both receive ZF precoding scheme with maximum-ratio transmission (MRT) scheme, and maximalratio combining (MRC) with transmit $\mathrm{ZF}$ scheme have been presented.

The performance analysis of the classical MIMO HD relaying systems, with multiple antennas at the source and destination, and/or the relay, have been studied extensively in the literature (see e.g., [15]-[18]). A new exact closedform expression for the outage probability of spatially correlated MIMO HD relaying systems, with multiple antennas at the source and destination applying the MRT/MRC scheme assuming perfect channel state information (CSI), has been derived in [16]. Moreover, in [15], the performance analysis of MIMO HD relaying beamforming is addressed, where an exact outage probability, approximate and upper-bound ergodic capacity expressions are derived. In [10], different spatial LI suppression techniques through MIMO FD relaying are presented, namely, antenna selection, beam selection, and null space projection.

More relevant to out work are [11], [19], where the outage probability and/or ergodic capacity expressions are derived. In [19], MIMO FD relaying systems and MIMO HD relaying systems' outage probability with antenna subset selection is considered. In [11], an exact closed-form and asymptotic outage probability expressions are addressed, where receive ZF/MRT scheme, MRC/transmit ZF scheme, and antenna selection are considered for MIMO FD relaying systems, with multiple antennas at the source and destination, spatially uncorrelated fading and perfect CSI. It is to be emphasized that all previously published work on MIMO FD relaying systems address the outage probability performance with spatially independent antennas and perfect CSI (see e.g., [7], [8], [11], [12], [19]-[23]). However, the joint impact of antenna correlation and imperfect CSI on the performance of MIMO FD relaying systems is of practical importance and has not been investigated yet.

Motivated by the above mentioned limitations and owing to the practical MIMO wireless transmission impairments, a spatial fading correlation exists due to the use of multiple transmit and receive antennas in a limited space, and also due to poor scattering conditions and limited angular spread. Meanwhile, perfect CSI is not available as channel estimation is performed in practice, hence, a residual desired and LI channels error exist due to imperfect CSI. Therefore, this paper investigates the impact of spatial fading correlation, and channel estimation errors for both the desired and LI channels, on the performance of MIMO FD relaying systems with receive $\mathrm{ZF} / \mathrm{MRT}$ scheme.

The main contributions of this paper are summarized as follows:

1) An exact closed-form outage probability expression is derived for spatially correlated MIMO FD relaying systems with arbitrary correlation matrix and in the presence of channel estimation errors, where receive $\mathrm{ZF} / \mathrm{MRT}$ scheme is applied to maximize the overall SNR at the FD relay.

2) A simpler special cases of the outage probability expression with exponential correlation matrix and independent antennas are also presented. In addition, a simpler tight closed-form lower-bound outage probability expressions are also included. Besides, the characterization of high SNR outage probability show that the achievable diversity order is $\min \left(N_{R}-1, N_{T}\right)$, where $N_{R}$ and $N_{T}$ are the number of relay receive and transmit antennas, respectively.

3) The presented analytical expressions efficiently evaluate the outage probability of MIMO FD relaying with receive $\mathrm{ZF} / \mathrm{MRT}$ scheme. Therefore, without resorting to the time consuming Monte Carlo simulations, the impact of some system key parameters such as the number of relay receive and transmit antennas, spatial correlation structure and coefficient, diversity order, estimation error variance, and the source transmit power on the system's outage probability are investigated.

The structure of the rest of the paper is as follows. In section II, we introduce the system model. In section III, the instantaneous overall SNR is addressed. In section IV, outage probability analysis is considered. Numerical results are provided in section V. Finally, section VI concludes the paper.

\section{The System Model}

Fig. 1 depicts the considered three node MIMO FD relaying network, where the source node $S$ is equipped with single antenna, and intends to transmit data to the destination node $D$ which is also equipped with single antenna via a MIMO 


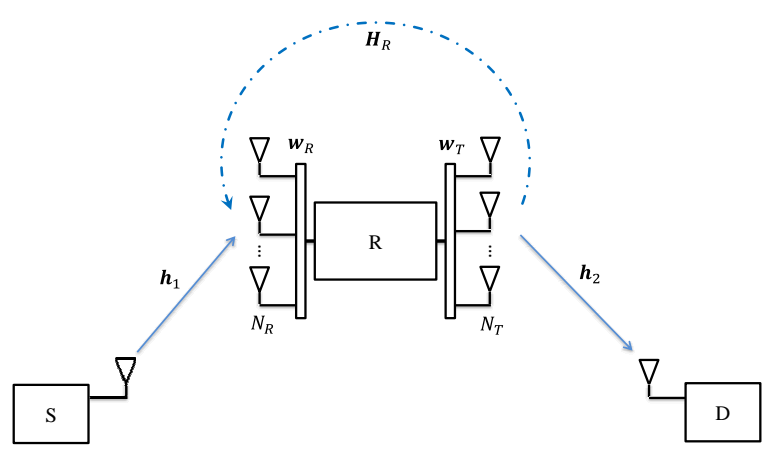

Figure 1: The MIMO FD relaying system model.

full-duplex AF relay $\boldsymbol{R}$. The relay is equipped with $N_{R}$ antennas for reception and $N_{T}$ antennas for transmission. It is assumed here that there is no direct link between the source and destination due to high path loss and heavy shadowing. Meanwhile, for comparison purposes, the case of MIMO HD relaying is also included with the constraint that the total number of antennas at the HD relay is $N=N_{R}+N_{T}$.

\section{A. Channel Model}

The statistical spatial correlation among the receiving and transmitting antennas, respectively, at the MIMO FD relay has to be characterized in practice due to space and/or scattering limitations. Therefore, the $\boldsymbol{S} \rightarrow \boldsymbol{R}$ channel, $\boldsymbol{h}_{1}$ and the $\boldsymbol{R} \rightarrow \boldsymbol{D}$ channel, $\boldsymbol{h}_{2}$ are subject to frequency flat correlated Rayleigh fading channel distributed according to $\boldsymbol{h}_{1} \sim$ $\mathcal{C N}(0, \boldsymbol{\Omega})$ with $\boldsymbol{\Omega}=\mathbb{E}\left[\boldsymbol{h}_{1} \boldsymbol{h}_{1}^{\dagger}\right]$ and $\boldsymbol{h}_{2} \sim \mathcal{C N}(0, \boldsymbol{\Lambda})$ with $\boldsymbol{\Lambda}=\mathbb{E}\left[\boldsymbol{h}_{2} \boldsymbol{h}_{2}^{\dagger}\right]$, respectively, where $\mathbb{E}(\cdot)$ is the expectation operator, and $\dagger$ denotes the conjugate transpose, assuming that the channel coherence time equals at least two time slots (this condition is necessary for the MIMO HD relaying systems case as MIMO FD relaying systems use only one time slot to transmit its information from the $S \rightarrow D$ ). The noise at the relay and destination are modeled by complex additive white Gaussian noise (AWGN) with zero mean and covariance of $\sigma_{n}^{2} \boldsymbol{I}$. In addition, $\boldsymbol{H}_{R}$ denotes the residual LI $\boldsymbol{R} \rightarrow \boldsymbol{R}$ channel, which is the resultant error due to imperfect LI mitigation performed by antennas isolation, analog and digital cancellation at the FD relay. Here, we model the residual LI channel $\boldsymbol{H}_{R}$ via independent Rayleigh fading channel distributed according to $\boldsymbol{H}_{R} \sim \mathcal{C N}(0, I)$. In order to further reduce the effect of residual LI at the MIMO FD relaying, receive $\mathrm{ZF}$ precoding scheme with MRT are performed. Note that this interference suppression and beamforming require an estimate of $\boldsymbol{h}_{1}, \boldsymbol{h}_{2}$, and $\boldsymbol{H}_{R}$ where channel estimation is required.

\section{B. Imperfect Channel Estimation}

In practice, a training sequence is used to estimate the channel. Let $\boldsymbol{x}$ and $\widehat{\boldsymbol{x}}$ denote the actual channel and the estimated channel, respectively. It is assumed that $\boldsymbol{x}$ and $\widehat{\boldsymbol{x}}$ are jointly ergodic and stationary Gaussian processes. In addition, it is also assumed that the channel estimate $\widehat{\boldsymbol{x}}$ and estimation error $\varepsilon$ are orthogonal. Therefore, in the case of imperfect channel estimation, the channel can be decomposed as

$$
\boldsymbol{x}=\widehat{\boldsymbol{x}}+\varepsilon
$$

where $\boldsymbol{x} \in\left\{\boldsymbol{h}_{1}, \boldsymbol{h}_{2}, \boldsymbol{H}_{R}\right\}$ denotes the true parameter value, $\widehat{\boldsymbol{x}} \in\left\{\widehat{\boldsymbol{h}}_{1}, \widehat{\boldsymbol{h}}_{2}, \widehat{\boldsymbol{H}}_{R}\right\}$ denotes the estimated parameter value, and $\varepsilon \in\left\{\varepsilon_{1}, \varepsilon_{2}, \varepsilon_{3}\right\}$ is the estimation error, for the $S \rightarrow$ $\boldsymbol{R}$ channel, $\boldsymbol{R} \rightarrow \boldsymbol{D}$ channel, and $\boldsymbol{R} \rightarrow \boldsymbol{R}$ self-interference channel, respectively, where $\varepsilon$ is the channel estimation error vector (matrix in the LI channel case), distributed according to $\varepsilon \sim \mathcal{C N}\left(0, \sigma_{\varepsilon}^{2} \boldsymbol{\Upsilon}\right)$, where $\boldsymbol{\Upsilon} \in\{\boldsymbol{\Omega}, \boldsymbol{\Lambda}, \boldsymbol{I}\}$. It is to be emphasized that the parameter $\sigma_{\varepsilon}^{2}$ captures the quality of the channel estimation, which is chosen depending on the used method of estimation, and depends on the training sequence length as well as the pilot power.

\section{The InStantaneous OVERALl SNR}

Following similar derivations to [11, Eq. (20)], and with the help of [24, Proposition 1], we arrive at the following overall SNR of MIMO full duplex relaying [25]

$$
\gamma=\frac{\gamma_{1} \gamma_{2}}{\gamma_{1}+\gamma_{2}+c}
$$

where $\gamma_{1}=\frac{\rho_{1}\left\|P \widehat{\boldsymbol{h}}_{1}\right\|^{2}}{\left(\rho_{1} \sigma_{\varepsilon_{1}}^{2} \frac{\left(P \widehat{\boldsymbol{h}}_{1}\right)^{\dagger} \boldsymbol{\Sigma}\left(P \widehat{\boldsymbol{h}}_{1}\right)}{\left\|P \widehat{\boldsymbol{h}}_{1}\right\|^{2}}+\rho_{2} \sigma_{\varepsilon_{3}}^{2}+1\right)}$, with $\rho_{1}=\frac{P_{S}}{\sigma_{n}^{2}}$ denotes the first-hop SNR and $P_{S}$ is the source average transmit power, $\gamma_{2}=\frac{\rho_{2}\left\|\widehat{\boldsymbol{h}}_{2}\right\|^{2}}{\left(\rho_{2} \sigma_{\varepsilon_{2}}^{2} \frac{\hat{h}_{2}^{\dagger} \Lambda \widehat{h}_{2}}{\left\|\widehat{h}_{2}\right\|^{2}}+1\right)}$, with $\rho_{2}=\frac{P_{R}}{\sigma_{n}^{2}}$ denotes the second-hop SNR and $P_{R}$ is the relay average transmit power, and $c=\frac{\rho_{2} \sigma_{\varepsilon_{2}}^{2} \frac{\widehat{\boldsymbol{h}}_{2}^{\dagger} \Lambda \widehat{h}_{2}}{\left\|\widehat{h}_{2}\right\|^{2}}\left(\rho_{1} \sigma_{\varepsilon_{1}}^{2} \frac{\left(P \widehat{\boldsymbol{h}}_{1}\right)^{\dagger} \boldsymbol{\Sigma}\left(P \widehat{\boldsymbol{h}}_{1}\right)}{\left\|P \widehat{h}_{1}\right\|^{2}}+\rho_{2} \sigma_{\varepsilon_{3}}^{2}+1\right)+1}{\left(\rho_{1} \sigma_{\varepsilon_{1}}^{2} \frac{\left(P \widehat{\boldsymbol{h}}_{1}\right)^{\dagger} \boldsymbol{\Sigma}\left(P \widehat{\boldsymbol{h}}_{1}\right)}{\left\|P \widehat{\boldsymbol{h}}_{1}\right\|^{2}}+\rho_{2} \sigma_{\varepsilon_{3}}^{2}+1\right)\left(\rho_{2} \sigma_{\varepsilon_{2}}^{2} \frac{\widehat{h}_{2}^{\dagger} \Lambda \widehat{h}_{2}}{\left\|\widehat{\boldsymbol{h}}_{2}\right\|^{2}}+1\right)}$. The projection matrix is given as $P=\boldsymbol{I}-\frac{\widehat{\boldsymbol{H}}_{R} \widehat{\boldsymbol{h}}_{2} \widehat{\boldsymbol{h}}_{2}^{\dagger} \widehat{\boldsymbol{H}}_{R}^{\dagger}}{\left\|\widehat{\boldsymbol{H}}_{R} \widehat{\boldsymbol{h}}_{2}\right\|^{2}}$, with $\|\cdot\|$ being the Frobenius norm.

As far as a simple closed-form outage probability expression is concern, equation (2) is mathematically intractable and does not lend itself to a closed-form outage probability expression. Therefore, in this paper, for simplicity and mathematical tractability, the estimation error covariance matrix is assumed to be distributed according to $\varepsilon \sim \mathcal{C N}\left(0, \sigma_{\varepsilon}^{2} \boldsymbol{I}\right)^{1}$. Hence, the resultant overall SNR is simplified to [26]

$$
\gamma=\frac{\gamma_{1} \gamma_{2}}{\gamma_{1}+\gamma_{2}+c}
$$

where $\gamma_{1}=\frac{\rho_{1}\left\|P \widehat{\boldsymbol{h}}_{1}\right\|^{2}}{\left(\rho_{1} \sigma_{\varepsilon_{1}}^{2}+\rho_{2} \sigma_{\varepsilon_{3}}^{2}+1\right)}, \gamma_{2}=\frac{\rho_{2}\left\|\widehat{\boldsymbol{h}}_{2}\right\|^{2}}{\left(\rho_{2} \sigma_{\varepsilon_{2}}^{2}+1\right)}$, and $c=$ $\frac{\rho_{2} \sigma_{\varepsilon_{2}}^{2}\left(\rho_{1} \sigma_{\varepsilon_{1}}^{2}+\rho_{2} \sigma_{\varepsilon_{3}}^{2}+1\right)+1}{\left(\rho_{1} \sigma_{\varepsilon_{1}}^{2}+\rho_{2} \sigma_{\varepsilon_{3}}^{2}+1\right)\left(\rho_{2} \sigma_{\varepsilon_{2}}^{2}+1\right)}$.

It is to be emphasized that in the case of perfect CSI (i.e., $\sigma_{\varepsilon}^{2}=0$ ) and/or independent fading channels (i.e., $\boldsymbol{\Omega}=\boldsymbol{\Lambda}=$ $\boldsymbol{I})$, Eq. (2) and Eq. (3) becomes identical and hence result in the same exact outage probability analysis.

The matrix $P$ is an idempotent orthogonal projection (nullspace projection) matrix, which is used to eliminate the loopback self-interference channel (i.e. the relay receives

\footnotetext{
${ }^{1}$ Note that this assumption is shown to be a tight lower-bound to the exact outage analysis of (2) in Fig. 3.
} 
and transmits on orthogonal subspaces). The vector $\widetilde{\boldsymbol{h}_{1}}=$ $P \widehat{\boldsymbol{h}}_{1}$ has the same statistics as $\widehat{\boldsymbol{h}}_{1}$ with dimensionality reduced by one [24], hence $\widetilde{\boldsymbol{h}_{1}} \sim \mathcal{C N}(0, \boldsymbol{\Sigma})$, where $\boldsymbol{\Sigma}=$ $\boldsymbol{\Omega}\left(1: N_{R}-1,1: N_{R}-1\right)$. For the ZF to be applicable in spatially correlated MIMO systems, channels need not be fully correlated (i.e., $\rho<1$ ). Note that for independent fading channels (i.e., $\boldsymbol{\Sigma}=\boldsymbol{\Lambda}=I$ ) with perfect CSI (i.e., $\left.\sigma_{\varepsilon_{i}}^{2}=0\right)^{2}$, equation (3) reduces to [11, Eq. (20)].

\section{Outage Probability Analysis}

In this section, the information outage probability of the proposed overall SNR of MIMO FD relaying systems is investigated. An exact as well as lower-bound expressions for the outage probability are derived for arbitrary correlation, where simpler correlation scenarios and lower bounds are also included. The outage probability is defined as the probability that the instantaneous mutual information, $\mathcal{I}=\log _{2}(1+\gamma)$, falls below a target rate of $R_{0}$ bits per channel use ${ }^{3}$. And can be given as

$$
\begin{aligned}
\mathrm{P}_{\text {out }}\left(R_{0}\right) & =\operatorname{Pr}\left(\log _{2}(1+\gamma)<R_{0}\right) \\
& =F_{\gamma}\left(\gamma_{T}\right) .
\end{aligned}
$$

where $\gamma_{T}=2^{R_{0}}-1$, and $F_{\gamma}(\cdot)$ denotes the cumulative distribution function (CDF) of the overall SNR.

\section{A. Arbitrary Correlation Case}

The CDF and the probability density function (PDF) of the random variables (RVs) $\gamma_{1}$ and $\gamma_{2}$, respectively, with arbitrary correlation matrix, can be written as [27]

$$
\begin{aligned}
F_{\gamma_{1}}(x)=1-\sum_{i=1}^{\varrho(\boldsymbol{\Sigma})} \sum_{j=1}^{\tau_{i}(\boldsymbol{\Sigma})} \sum_{k=0}^{j-1} \frac{\chi_{i, j}(\boldsymbol{\Sigma})}{k !} \\
\quad \times\left(\frac{x}{\overline{\gamma_{1}} \alpha_{\langle i\rangle}}\right)^{k} e^{-\frac{x}{\overline{\gamma_{1}} \alpha\langle i\rangle}}, \quad x \geq 0
\end{aligned}
$$

and

$$
\begin{aligned}
& f_{\gamma_{2}}(y)=\sum_{l=1}^{\varrho(\boldsymbol{\Lambda})} \sum_{m=1}^{\tau_{l}(\boldsymbol{\Lambda})} \chi_{l, m}(\boldsymbol{\Lambda}) \frac{\left(\overline{\gamma_{2}} \beta_{\langle l\rangle}\right)^{-m}}{\Gamma(m)} \\
& \times y^{m-1} e^{-\frac{y}{\overline{\gamma_{2} \beta}\langle l\rangle}}, \quad y \geq 0
\end{aligned}
$$

where $\boldsymbol{\Sigma}$ is the correlation matrix of $\widetilde{\boldsymbol{h}_{1}}$ with eigenvalues $\alpha_{1}, \alpha_{2}, \ldots, \alpha_{N_{R}-1}$ in any order, $\varrho(\boldsymbol{\Sigma})$ is the number of distinct eigenvalues of $\boldsymbol{\Sigma}, \alpha_{\langle 1\rangle}>\alpha_{\langle 2\rangle}>\ldots>\alpha_{\langle\varrho(\boldsymbol{\Sigma})\rangle}$ are the distinct eigenvalues in decreasing order, $\tau_{i}(\boldsymbol{\Sigma})$ is the multiplicity of $\alpha_{\langle i\rangle}, \chi_{i, j}(\boldsymbol{\Sigma})$ is the $(i, j)^{\text {th }}$ characteristic function of $\boldsymbol{\Sigma}$ [27, Eq. (129)]. Similarly, $\boldsymbol{\Lambda}$ is the correlation matrix of $\boldsymbol{h}_{2}$ with eigenvalues $\beta_{1}, \beta_{2}, \ldots, \beta_{N_{T}}$ in any order, $\varrho(\boldsymbol{\Lambda})$ is the number of distinct eigenvalues of $\boldsymbol{\Lambda}, \beta_{\langle 1\rangle}>$

\footnotetext{
${ }^{2}$ It is to be emphasized that $c$ can be accurately approximated to 1 , this has been proved in [25].

${ }^{3}$ Note that in contrast to (4), the SNR outage probability can be defined as the probability that the instantaneous overall $\gamma$, falls below a threshold $\gamma_{T}$; $\operatorname{Pr}\left(\gamma<\gamma_{T}\right)=F_{\gamma}\left(\gamma_{T}\right)$. Note that according to (4), $\gamma_{T}$ in the case of HD relaying is given as $\gamma_{T}=2^{2 R_{0}}-1$.
}

$\beta_{\langle 2\rangle}>\ldots>\beta_{\langle\varrho(\boldsymbol{\Lambda})\rangle}$ are the distinct eigenvalues in decreasing order, $\tau_{l}(\boldsymbol{\Lambda})$ is the multiplicity of $\beta_{\langle l\rangle}$, and $\chi_{l, m}(\boldsymbol{\Lambda})$ is the $(l, m)^{\text {th }}$ characteristic function of $\boldsymbol{\Lambda}$.

1) Exact Outage Probability: The CDF of the overall SNR can be derived as [15, Appendix I]

$$
\begin{aligned}
& F_{\gamma}\left(\gamma_{T}\right)=\operatorname{Pr}\left(\frac{\gamma_{1} \gamma_{2}}{\gamma_{1}+\gamma_{2}+c}<\gamma_{T}\right) \\
& =1-\int_{0}^{\infty} \bar{F}_{\gamma_{1}}\left(\frac{\gamma_{T}\left(\gamma_{T}+w+c\right)}{w}\right) f_{\gamma_{2}}\left(\gamma_{T}+w\right) d w
\end{aligned}
$$

where $\bar{F}_{\gamma_{1}}(\cdot)$ is the complementary CDF of $\gamma_{1}$.

Theorem 1. The exact outage probability of the overall SNR $\gamma$, with arbitrary correlation matrices, can be derived as given in (8), shown at the top of next page.

where $\mathcal{K}_{v}(z)$ is the modified bessel function of the second kind of order $v$.

Proof: The proof is given in Appendix A.

2) Lower-Bound Outage Probability: The overall SNR can be upper bounded by ${ }^{4}$

$$
\gamma \leq \gamma_{\text {up }}=\min \left(\gamma_{1}, \gamma_{2}\right)
$$

Note that the overall SNR upper-bound for AF relaying systems (9) substitutes the exact overall SNR for the decodeand-forward (DF) relaying systems.

Therefore, the outage probability of the overall $\operatorname{SNR} \gamma$, with arbitrary correlation matrices, is lower-bounded by

$$
\begin{aligned}
F_{\gamma_{u p}}\left(\gamma_{T}\right)=1 & -\sum_{i=1}^{\varrho(\boldsymbol{\Sigma})} \sum_{j=1}^{\tau_{i}(\boldsymbol{\Sigma})} \chi_{i, j}(\boldsymbol{\Sigma}) \frac{\Gamma\left(j, \frac{\gamma_{T}}{\overline{\gamma_{1}} \alpha_{\langle i\rangle}}\right)}{\Gamma(j)} \\
& \times \sum_{k=1}^{\varrho(\boldsymbol{\Lambda})} \sum_{l=1}^{\tau_{k}(\boldsymbol{\Lambda})} \chi_{k, l}(\boldsymbol{\Lambda}) \frac{\Gamma\left(l, \frac{y}{\overline{\gamma_{2}} \beta_{\langle k\rangle}}\right)}{\Gamma(l)} .
\end{aligned}
$$

Proof: The proof is given in Appendix B.

\section{B. Exponential Correlation Case}

In the case of exponential correlation matrix, all eigenvalues are distinct with multiplicity of one. Therefore, $\varrho(\Sigma)=N_{R}-$ $1, \tau_{i}(\boldsymbol{\Sigma})=1, \varrho(\boldsymbol{\Lambda})=N_{T}, \tau_{m}(\boldsymbol{\Lambda})=1$. Hence, the simplified CDF and PDF of $\gamma_{1}$ and $\gamma_{2}$, respectively, given as

$$
F_{\gamma_{1}}(x)=1-\sum_{i=1}^{N_{R}-1} \chi_{i}(\boldsymbol{\Sigma}) e^{-\frac{x}{\gamma_{1} \alpha_{i}}}
$$

and

$$
f_{\gamma_{2}}(y)=\sum_{j=1}^{N_{T}} \frac{\chi_{j}(\boldsymbol{\Lambda})}{\overline{\gamma_{2}} \beta_{j}} e^{-\frac{y}{\gamma_{2} \beta_{j}}}
$$

where $\chi_{i}(\boldsymbol{\Sigma})=\alpha_{i}^{N_{R}-2} \prod_{k=1, k \neq i}^{N_{R}-1}\left(\alpha_{i}-\alpha_{k}\right)^{-1}$, and $\chi_{j}(\boldsymbol{\Lambda})=\beta_{j}^{N_{T}-1} \prod_{n=1, k \neq j}^{N_{T}}\left(\beta_{j}-\beta_{n}\right)^{-1}$.

\footnotetext{
${ }^{4}$ It is well known in the conventional half-duplex relaying literature that the overall SNR $\gamma=\frac{\gamma_{1} \gamma_{2}}{\gamma_{1}+\gamma_{2}+1}$ can be tightly upper-bounded by $\frac{\gamma_{1} \gamma_{2}}{\gamma_{1}+\gamma_{2}}$ (see e.g., [28, Eq. (6)]). In addition, the upper-bound $\frac{\gamma_{1} \gamma_{2}}{\gamma_{1}+\gamma_{2}}$ can be further upperbounded by $\min \left(\gamma_{1}, \gamma_{2}\right)$ (see e.g., [29, Eq. (8)]). It is to be emphasized that the asymptotic results of these bounds are exact.
} 


$$
\begin{aligned}
F_{\gamma}\left(\gamma_{T}\right)=1-2 \sum_{i=1}^{\varrho(\boldsymbol{\Sigma})} & \sum_{j=1}^{\tau_{i}(\boldsymbol{\Sigma})} \sum_{k=0}^{j-1} \frac{\chi_{i, j}(\boldsymbol{\Sigma})}{k !}\left(\frac{\gamma_{T}}{\overline{\gamma_{1}} \alpha_{\langle i\rangle}}\right)^{k} e^{-\frac{\gamma_{T}}{\overline{\gamma_{1}} \alpha_{\langle i\rangle}}} \sum_{l=0}^{k}\left(\begin{array}{c}
k \\
l
\end{array}\right)\left(\gamma_{T}+c\right)^{l} \sum_{m=1}^{\varrho(\boldsymbol{\Lambda})} \sum_{n=1}^{\tau_{m}(\boldsymbol{\Lambda})} \chi_{m, n}(\boldsymbol{\Lambda}) \frac{1}{\Gamma(n)} e^{-\frac{\gamma_{T}}{\overline{\gamma_{2}} \beta\langle m\rangle}} \\
& \times \sum_{p=0}^{n-1}\left(\begin{array}{c}
n-1 \\
p
\end{array}\right) \gamma_{T}^{n-p-1}\left(\overline{\gamma_{2}} \beta_{\langle m\rangle}\right)^{p-n-l+1}\left(\frac{\gamma_{T}^{2}+\gamma_{T} c}{\overline{\gamma_{1} \gamma_{2}} \alpha_{\langle i\rangle} \beta_{\langle m\rangle}}\right)^{\frac{p-l+1}{2}} \mathcal{K}_{p-l+1}\left(2 \sqrt{\frac{\gamma_{T}^{2}+\gamma_{T} c}{\overline{\gamma_{1} \gamma_{2}} \alpha_{\langle i\rangle} \beta_{\langle m\rangle}}}\right)
\end{aligned}
$$

1) Exact Outage Probability: The exact outage probability of the overall SNR $\gamma$, with exponential correlation matrices, can be written as

$$
\begin{aligned}
F_{\gamma}\left(\gamma_{T}\right)=1- & 2 \sum_{i=1}^{N_{R}-1} \chi_{i}(\boldsymbol{\Sigma}) e^{-\frac{\gamma_{T}}{\gamma_{1} \alpha_{i}}} \sum_{j=1}^{N_{T}} \chi_{j}(\boldsymbol{\Lambda}) e^{-\frac{\gamma_{T}}{\gamma_{2} \beta_{j}}} \\
& \times \sqrt{\frac{\gamma_{T}^{2}+\gamma_{T} c}{\overline{\gamma_{1} \gamma_{2}} \alpha_{i} \beta_{j}}} \mathcal{K}_{1}\left(2 \sqrt{\frac{\gamma_{T}^{2}+\gamma_{T} c}{\overline{\gamma_{1} \gamma_{2}} \alpha_{i} \beta_{j}}}\right)
\end{aligned}
$$

Proof: The proof is given in Appendix C.

2) Lower-Bound Outage Probability: The outage probability of the overall SNR $\gamma$, with exponential correlation matrices, is lower-bounded by

$$
F_{\gamma_{\text {up }}}\left(\gamma_{T}\right)=1-\sum_{i=1}^{N_{R}-1} \chi_{i}(\boldsymbol{\Sigma}) e^{-\frac{\gamma_{T}}{\gamma_{1} \alpha_{i}}} \sum_{j=1}^{N_{T}} \chi_{j}(\boldsymbol{\Lambda}) e^{-\frac{\gamma_{t h}}{\bar{\gamma}_{2} \beta_{j}}}
$$

Proof: Following similar steps to the derivations of (10), the outage probability of the upper-bound overall SNR can be derived by substituting the CDF of $\gamma_{1}$ and $\gamma_{2}$ into (25) which upon tedious simplification reduces to (14).

3) Asymptotic Analysis: In the asymptotic high SNR regime, the asymptotic outage probability of the overall SNR $\gamma$ can be expressed as

$$
\begin{array}{r}
F_{\gamma}^{\infty}\left(\gamma_{T}\right)=\frac{\left(\frac{\gamma_{T}}{\gamma_{1}}\right)^{N_{R}-1}}{\prod_{i=0}^{N_{R}-1} \alpha_{i} \Gamma\left(N_{R}\right)}+\frac{\left(\frac{\gamma_{T}}{\bar{\gamma}_{1}}\right)^{N_{T}}\left(\frac{1}{\kappa}\right)^{N_{T}}}{\prod_{i=0}^{N_{T}} \beta_{i} \Gamma\left(N_{T}+1\right)} \\
+O\left(\left(\frac{\gamma_{T}}{\overline{\gamma_{1}}}\right)^{\min \left(N_{R}, N_{T}+1\right)}\right) .
\end{array}
$$

Proof: The proof follow similar steps to the derivation of [17, Eq. (17)].

It is easily seen from the above asymptotic expression that the achievable diversity order of correlated MIMO FD relaying systems with ZF/MRT scheme is $\min \left(N_{R}-1, N_{T}\right)$. Furthermore, the negative impact of correlation on the outage probability is clearly seen here, where the outage probability increase is quantified by the factors $\frac{1}{\prod_{i=0}^{N_{R}-1} \alpha_{i}}$ and $\frac{1}{\prod_{i=0}^{N_{T}} \beta_{i}}$. Meanwhile, the outage probability increase due to estimation error is determined by the factors $\left(\frac{\rho_{1} \sigma_{\varepsilon_{1}}^{2}+\rho_{2} \sigma_{\varepsilon_{3}}^{2}+1}{1-\sigma_{\varepsilon_{1}}^{2}}\right)^{N_{R}-1}$ and $\left(\frac{\rho_{2} \sigma_{\varepsilon_{2}}^{2}+1}{1-\sigma_{\varepsilon_{2}}^{2}}\right)^{N_{T}}$.

\section{Independent Case}

In the case of independent channels, the CDF and PDF of the RVs $\gamma_{1}$ and $\gamma_{2}$ are (respectively) given as

$$
F_{\gamma_{1}}(x)=1-e^{-\frac{x}{\gamma_{1}}} \sum_{k=0}^{N_{R}-2} \frac{1}{k !}\left(\frac{x}{\overline{\gamma_{1}}}\right)^{k}, x \geq 0
$$

and

$$
f_{\gamma_{2}}(y)=\frac{{\overline{\gamma_{2}}}^{-N_{T}}}{\left(N_{T}-1\right) !} y^{N_{T}-1} e^{-\frac{y}{\gamma_{2}}}, y \geq 0 .
$$

1) Exact Outage Probability: The exact outage probability of the overall SNR $\gamma$, with independent channels, can be derived as in (18), shown at the top of next page.

Proof: The proof follow similar steps to the derivation of (8).

2) Lower-Bound Outage Probability: The outage probability of the overall SNR $\gamma$, with independent channels, is lowerbounded by

$$
F_{\gamma_{\text {up }}}\left(\gamma_{T}\right)=1-\frac{\Gamma\left(N_{R}-1, \frac{\gamma_{T}}{\gamma_{1}}\right)}{\Gamma\left(N_{R}-1\right)} \frac{\Gamma\left(N_{T}, \frac{\gamma_{T}}{\gamma_{2}}\right)}{\Gamma\left(N_{T}\right)} .
$$
(10).

Proof: The proof follow similar steps to the derivation of

3) Asymptotic Analysis: To validate and characterize the achievable diversity order of the MIMO full-duplex relaying system receive ZF/MRT scheme at the AF relay, we approximate (19) in the asymptotic high SNR regime where $\overline{\gamma_{2}}=\kappa \overline{\gamma_{1}}$, with $\kappa$ denotes a finite constant number and $\overline{\gamma_{1}} \rightarrow \infty$. Hence, the asymptotic outage probability of the overall SNR $\gamma$ can be expressed as

$$
\begin{aligned}
F_{\gamma}^{\infty}\left(\gamma_{T}\right)=\frac{\left(\frac{\gamma_{T}}{\bar{\gamma}_{1}}\right)^{N_{R}-1}}{\Gamma\left(N_{R}\right)}+\frac{\left(\frac{\gamma_{T}}{\bar{\gamma}_{1}}\right)^{N_{T}}}{\Gamma\left(N_{T}+1\right)}\left(\frac{1}{\kappa}\right)^{N_{T}} \\
+O\left(\left(\frac{\gamma_{T}}{\overline{\gamma_{1}}}\right)^{\min \left(N_{R}, N_{T}+1\right)}\right) .
\end{aligned}
$$

Proof: The proof is given in Appendix D.

Equation (20) can be further simplified to

$$
F_{\gamma}^{\infty}\left(\gamma_{T}\right)= \begin{cases}\frac{\left(\frac{\gamma_{T}}{\gamma_{1}}\right)^{N_{R}-1}}{\Gamma\left(N_{R}\right)} & , N_{R}-1<N_{T} \\ \frac{\left(\frac{\gamma_{T}}{\gamma_{1}}\right)^{N_{R}-1}}{\Gamma\left(N_{R}\right)}+\frac{\left(\frac{\gamma_{T}}{\gamma_{2}}\right)^{N_{T}}}{\Gamma\left(N_{T}+1\right)} & , N_{R}-1=N_{T} . \\ \frac{\left(\frac{\gamma_{T}}{\gamma_{2}}\right)^{N_{T}}}{\Gamma\left(N_{T}+1\right)} & , N_{R}-1>N_{T}\end{cases}
$$

It is straight forward to show from (21) that the achievable diversity order of the ZF/MRT MIMO FD relaying systems is 


$$
\begin{aligned}
F_{\gamma}\left(\gamma_{T}\right)=1-2 \frac{\gamma_{T}^{N_{T}-\frac{1}{2}} \sqrt{\gamma_{T}+c}}{\left(N_{T}-1\right) !{\overline{\gamma_{2}^{\mathrm{X}}}}^{N_{T}^{\mathrm{X}}}} \sqrt{\frac{\overline{\gamma_{2}}}{\bar{\gamma}_{1}}} e^{-\frac{\gamma_{T}}{\bar{\gamma}_{1}}-\frac{\gamma_{T}}{\bar{\gamma}_{2}}} \sum_{k=0}^{N_{R}-2} \frac{1}{k !}\left(\frac{\gamma_{T}}{\overline{\gamma_{1}}}\right)^{k} \sum_{l=0}^{k}\left(\begin{array}{c}
k \\
l
\end{array}\right) \\
\times\left(\frac{\overline{\gamma_{1}}\left(\gamma_{T}+c\right)}{\overline{\gamma_{2}} \gamma_{T}}\right)^{\frac{l}{2}} \sum_{m=0}^{N_{T}-1}\left(\begin{array}{c}
N_{T}-1 \\
m
\end{array}\right)\left(\frac{\gamma_{T}+c}{\overline{\gamma_{1}} \gamma_{T}} \overline{\gamma_{2}}\right)^{\frac{m}{2}} \mathcal{K}_{m-l+1}\left(2 \sqrt{\frac{\gamma_{T}^{2}+\gamma_{T} c}{\overline{\gamma_{1} \gamma_{2}}}}\right) .
\end{aligned}
$$

$\min \left(N_{R}-1, N_{T}\right)$. Therefore, correlation has no impact on the diversity order of the system.

\section{Hybrid Relaying Modes}

The case of MIMO half-duplex relaying with maximumratio combining (MRC)/MRT scheme [25, Eq. (5)] is considered with the constraint that the total number of antennas at the HD relay is $N=N_{R}+N_{T}$. The need for proper mode selection is motivitaed by Figs. 4-5. The outperform region of FD relaying can be derived by solving $\mathrm{P}_{\text {out }}^{\mathrm{FD}}\left(R_{0}\right)-\mathrm{P}_{\text {out }}^{\mathrm{HD}}\left(R_{0}\right) \leq 0$. Note that the outage probability expression for a hybrid relaying mode that switches to the appropriate mode according to the instantaneous CSI is given by

$$
\mathrm{P}_{\text {out }}^{\mathrm{Hybrid}}\left(R_{0}\right)=\operatorname{Pr}\left(\max \left(C_{\text {Inst }}^{\mathrm{FD}}, C_{\text {Inst }}^{\mathrm{HD}}\right)<R_{0}\right)
$$

where $C_{\text {Inst }}^{\mathrm{FD}}=\log _{2}\left(1+\gamma^{\mathrm{FD}}\right), C_{\text {Inst }}^{\mathrm{HD}}=\log _{2}\left(\sqrt{1+\gamma^{\mathrm{HD}}}\right)$, $\gamma^{\mathrm{FD}}$ is given in (3), and $\gamma^{\mathrm{HD}}$ is given in [25, Eq. (5)].

\section{Numerical Results}

In this section, we analyze and validate the presented theoretical results with Monte Carlo simulations. Without loss of generality, two common correlation scenarios are considered here; the uniform correlation case where $\boldsymbol{\Sigma}(i, j)=$ $\left\{\begin{array}{ll}1 & i=j \\ \rho & i \neq j\end{array}\right.$, and the exponential correlation case where $\boldsymbol{\Sigma}(i, j)=\rho^{|i-j|}$, with $\rho \in[0,1)$ being the correlation coefficient. For simplicity, symmetric settings $\left(\rho_{1}=\rho_{2}\right)$ are assumed for both links, with a target rate of $R_{0}=2$ bits per channel use. The Cholesky decomposition method is used to transform a set of uncorrelated Gaussian random vector to a set of correlated random vector of a predefined correlation matrix ${ }^{6}$. The correlation coefficient is defined using the practical channel model given in [30], assuming that there is a uniform linear antenna arrays at the relay. Let $d$ be the equidistant antenna spacing (spacing between adjacent antennas), normalized by the carrier wavelength, denoted as $d_{r}$ at the receiving antennas, and $d_{t}$ at the transmitting antennas

\footnotetext{
${ }^{5}$ Note that as far as a closed-form outage probability expression is concern the analysis of such hybrid relaying mode is a challenging mathematical problem due to the presence of high correlation between $\gamma^{\mathrm{HD}}$ and $\gamma^{\mathrm{FD}}$.

${ }^{6}$ The Cholesky decomposition of the predefined correlation matrix $\mathbf{\Upsilon}$ may be derived as: $\Upsilon=U \Psi U^{\dagger}=(U \sqrt{\Psi})(U \sqrt{\Psi})^{\dagger}$, where $U$ is a unitry eigenvector matrix and $\Psi$ is a diagonal matrix whose entries are the eigenvalues of $\boldsymbol{\Upsilon}$. Therefore, a spatially correlated channel random vector $\boldsymbol{y}$ is generated by $\boldsymbol{y}=L \boldsymbol{x}$, where $\boldsymbol{x}$ is a vector of independent random variables distributed according to $\boldsymbol{x} \sim \mathcal{C N}(0, I)$ and $L=U \sqrt{\Psi}$. Therefore,

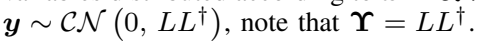

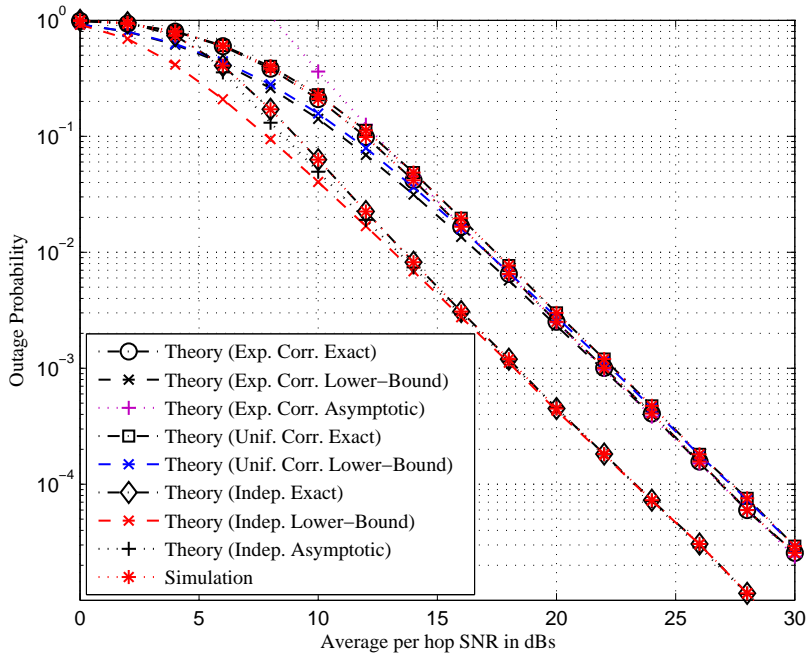

Figure 2: MIMO FD relaying outage probability with perfect CSI $\left(\sigma_{\varepsilon}^{2}=0\right)$ against the first hop $\operatorname{SNR}\left(\rho_{1}\right)$ where $\rho_{1}=\rho_{2}$, for the case of high $\left(\sigma_{\theta_{r}}^{2}=\sigma_{\theta_{t}}^{2}=\frac{\pi}{64}, d_{r}=d_{t}=\frac{1}{3}\right)$ uniform, exponential and no correlations, and $N_{R}=N_{T}=3$.

( $d_{r}=d_{t}$ in the MIMO HD relaying case). Two cases for $d$ are assumed here; the fixed antenna spacing case where $d$ is fixed regardless of the chosen number of antennas, and the variable $d$ case where the inter-antenna spacing is changing according to the limited available space and the chosen number of antennas put in that space, hence $d=\frac{D}{N-1}$, where $D$ is the limited multi-antenna space at the relay, normalized by the carrier wavelength. Also, let $\bar{\theta}_{r}$ and $\bar{\theta}_{t}$ be the mean angle or arrival (AoA) and mean angle of departure (AoD), respectively. In addition, let $\sigma_{\theta_{r}}^{2}$ and $\sigma_{\theta_{t}}^{2}$ as the receive and transmit cluster angle spread, respectively. Assuming that the actual AoA and AoD may be given by $\theta_{r}=\bar{\theta}_{r}+\widehat{\theta}_{r}$ and $\theta_{t}=\bar{\theta}_{t}+\widehat{\theta}_{t}$, respectively, with $\widehat{\theta}_{r} \sim \mathcal{C N}\left(0, \sigma_{\theta_{r}}^{2}\right)$ and $\widehat{\theta}_{t} \sim \mathcal{C N}\left(0, \sigma_{\theta_{t}}^{2}\right)$. Moreover, The receive and transmit antennas correlation matrices are defined as $\boldsymbol{\Sigma}=f\left(\rho\left(d_{r}, \bar{\theta}_{r}, \sigma_{\theta_{r}}^{2}\right)\right)$ and $\boldsymbol{\Lambda}=f\left(\rho\left(d_{t}, \bar{\theta}_{t}, \sigma_{\theta_{t}}^{2}\right)\right)$, respectively, where the function $f(\cdot)$ used to differentiate between different correlation scenarios (i.e., the defined uniform and exponential correlation). With these assumptions, the correlation coefficient $\rho$ can be estimated as

$$
\rho\left(d, \bar{\theta}, \sigma_{\theta}^{2}\right)=e^{-j 2 \pi d \cos (\bar{\theta})} e^{-\frac{1}{2}\left(2 \pi d \sin (\bar{\theta}) \sigma_{\theta}\right)^{2}} .
$$

Which implies that large antenna spacing and/or large cluster angle spread results in small spatial correlation and vice versa. For all results in this section, it is assumed that $\bar{\theta}_{r}=\bar{\theta}_{t}=\frac{\pi}{2}$. 


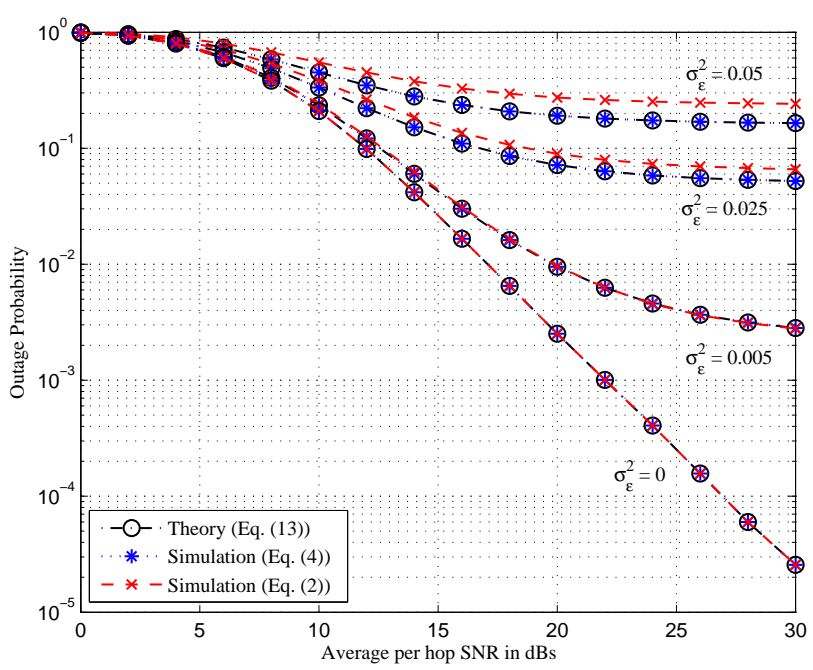

Figure 3: MIMO FD relaying outage probability with imperfect CSI against the first hop SNR $\left(\rho_{1}\right)$ where $\rho_{1}=\rho_{2}$, for the case of high $\left(\sigma_{\theta_{r}}^{2}=\sigma_{\theta_{t}}^{2}=\frac{\pi}{64}, d_{r}=d_{t}=\frac{1}{3}\right)$ exponential and no correlations, and $N_{R}=N_{T}=3$.

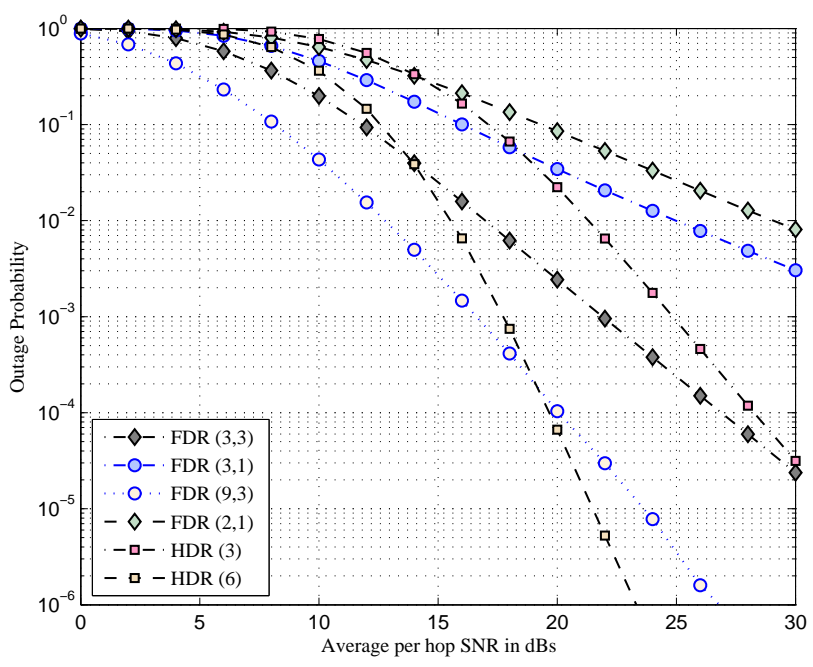

Figure 4: MIMO relaying outage with perfect CSI probability against the first hop SNR $\left(\rho_{1}\right)$ where $\rho_{1}=\rho_{2}$, for high exponential correlation $\left(\sigma_{\theta_{r}}^{2}=\sigma_{\theta_{t}}^{2}=\frac{\pi}{64}, d_{r}=d_{t}=\frac{1}{3}\right)$.

In Fig. 2, the outage probability of MIMO FD relaying against the first-hop SNR is presented with different correlation matrix scenarios, where Monte Carlo simulations of (4) is used to validate the new closed-form analytical expressions (8), (13), and (18). It is seen that the simulation and proposed analytical expression provide a perfect match which corroborate the exactness of the proposed exact closedform analytical expressions. In addition, the tightness of the proposed lower-bound and asymptotic expressions are also verified. The chosen values of the number of relay receive and transmit antennas in the FD relaying case are shown as $\left(N_{R}, N_{T}\right)$, where in the HD relaying is shown as $(N)$. Fig. 3

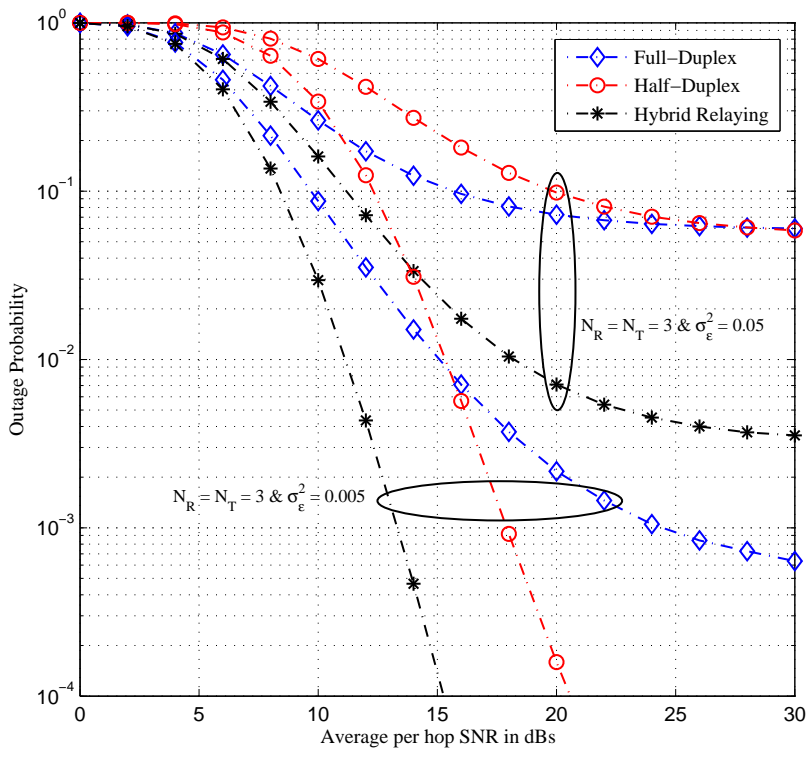

Figure 5: MIMO FD, HD, and hybrid relaying outage probability with imperfect CSI against the first hop SNR $\left(\rho_{1}\right)$ where $\rho_{1}=\rho_{2}$,for exponential correlation with $\sigma_{\theta_{r}}^{2}=\sigma_{\theta_{t}}^{2}=\frac{\pi}{64}$, $d_{r}=d_{t}=\frac{2}{N_{X}-1}$, where $N_{X}=N_{R}=N_{T}$ in the FD relaying case, and $N_{X}=N_{R}+N_{T}=N$ in the HD relaying case, with different $\sigma_{\varepsilon}^{2}$.

shows the effect of imperfect CSI on the MIMO FD relaying outage probability in the case of fixed antennas spacing and exponential correlation matrix. The tightness of the approximation of the estimation error covariance is also verified. A higher outage probability degradation is seen as the estimation error variance $\sigma_{\varepsilon}^{2}$ increases, where in the presence of imperfect CSI an error floor is seen at high SNR. In Fig. 4, the outage probability of MIMO FD relaying with receive ZF/MRT scheme is shown with different antenna configurations, where MIMO HD relaying with MRC/MRT is also presented. For instance, once comparing the FD relaying settings $(3,3)$ with the HD relaying of (6) and (3) for the separate and shared FD relaying antenna system configurations. It is seen that FD relaying outperforms the conventional HD relaying in the practical SNR range. Furthermore, a performance improvement is seen as a result of higher diversity order and/or array gain. For simplicity, it is assumed that $\sigma_{\varepsilon_{1}}^{2}=\sigma_{\varepsilon_{2}}^{2}=\sigma_{\varepsilon_{3}}^{2}=\sigma_{\varepsilon}^{2}$. Fig. 5 shows the impact of imperfect CSI on MIMO FD relaying, HD relaying, and hybrid relaying outage probability ${ }^{7}$ with variable antennas spacing. It is to be emphasized here that the degradation in outage probability due to spatial correlation is higher in MIMO HD relaying compared to MIMO FD relaying systems since higher number of antennas are planted on the same limited space. Meanwhile, the degradation in outage probability due to estimation error is higher in the MIMO FD relaying systems owing to the addition of the estimation error of the loopback self-interference channel. Therefore, for optimum performance, hybrid relaying modes is proposed

\footnotetext{
${ }^{7}$ Note that a lower bound outage probability for the hybrid relaying case is shown in Fig. 5 where $\gamma^{\mathrm{HD}}$ and $\gamma^{\mathrm{FD}}$ are assumed independent.
} 
which switches between HD and FD relaying modes.

\section{CONCLUSIONS}

In this paper, new closed-form expressions for the outage probability of spatially correlated MIMO FD relaying with receive ZF/MRT are presented, where simpler lower bounds are also included. The case of imperfect CSI is also addressed. Numerical results demonstrated that FD relaying systems outperforms HD relaying systems at low to medium SNR. In addition, in the presence of channel estimation errors, an outage probability error floor is seen as SNR increases. Besides, The performance degradation due to imperfect CSI is higher in the FD relaying case because of the extra LI channel estimation errors. Furthermore, although the source and destination are equipped with one antenna, the system performance can be improved by appropriately choosing the precoding scheme (receive ZF/MRT or MRC/transmit ZF). For superior system performance, receive $\mathrm{ZF}$ is performed when $N_{R}>N_{T}$, and transmit $\mathrm{ZF}$ is performed when $N_{R}<N_{T}$. However, when $N_{R}=N_{T}$, both receive $\mathrm{ZF}$ and transmit $\mathrm{ZF}$ have the same performance assuming symmetric settings $\left(\rho_{1}=\rho_{2}\right)$ case.

\section{APPENDIX A PROOF OF THEOREM 1}

From (7), it can be shown that the CDF of the overall SNR is given as (24), shown at the top of next page.

Where with the aid of [31, Eq. (3.471.9)], we arrive at (8), which concludes the proof.

\section{APPENDIX B PROOF OF EQUATION (10)}

From the overall SNR upper-bound (9), we have

$$
\begin{aligned}
F_{\gamma_{\text {up }}}\left(\gamma_{T}\right) & =\operatorname{Pr}\left(\min \left(\gamma_{1}, \gamma_{2}\right)<\gamma_{T}\right) \\
& =1-\left(1-F_{\gamma_{1}}\left(\gamma_{T}\right)\right)\left(1-F_{\gamma_{2}}\left(\gamma_{T}\right)\right)
\end{aligned}
$$

which upon substituting the CDF of $\gamma_{1}$ and $\gamma_{2}$ reduces to (10), that concludes the proof.

\section{APPENDIX C PROOF OF EQUATION (13)}

From (7), the CDF of the overall SNR can be derived as

$$
\begin{array}{r}
F_{\gamma}\left(\gamma_{T}\right)=1-\sum_{i=1}^{N_{R}-1} \chi_{i}(\boldsymbol{\Sigma}) \sum_{j=1}^{N_{T}} \frac{\chi_{j}(\boldsymbol{\Lambda})}{\beta_{j}} e^{-\frac{\gamma_{T}}{\gamma_{1} \alpha_{i}}-\frac{\gamma_{T}}{\gamma_{2} \beta_{j}}} \\
\times \int_{0}^{\infty} e^{-\frac{\gamma_{T}^{2}+\gamma_{T} c}{w \overline{\gamma_{1}} \alpha_{i}}} e^{-\frac{w}{\overline{\gamma_{2}} \beta_{j}}} d w
\end{array}
$$

Which with the aid of [31, Eq. (3.471.9)], reduces to (13), that concludes the proof.

\section{APPENDIX D \\ PROOF OF EQUATION (20)}

The asymptotic results can be easily obtained once invoking the asymptotic expansion of the incomplete gamma function [31, Eq. (8.354.1)]. Hence, in the high SNR regime where $\overline{\gamma_{2}}=\kappa \overline{\gamma_{1}}$ and $\overline{\gamma_{1}} \rightarrow \infty$, we have

$$
\gamma\left(N_{R}-1, \frac{\gamma_{T}}{\overline{\gamma_{1}}}\right)=\frac{\left(\frac{\gamma_{T}}{\overline{\gamma_{1}}}\right)^{N_{R}-1}}{N_{R}-1}+O\left(\left(\frac{\gamma_{T}}{\overline{\gamma_{1}}}\right)^{N_{R}}\right) .
$$

where $\gamma\left(N_{R}-1, \frac{\gamma_{T}}{\gamma_{1}}\right)+\Gamma\left(N_{R}-1, \frac{\gamma_{T}}{\gamma_{1}}\right)=\Gamma\left(N_{R}-1\right)$. Therefore,

$$
\frac{\Gamma\left(N_{R}-1, \frac{\gamma_{T}}{\gamma_{1}}\right)}{\Gamma\left(N_{R}-1\right)}=1-\frac{\left(\frac{\gamma_{T}}{\gamma_{1}}\right)^{N_{R}-1}}{\Gamma\left(N_{R}\right)}+O\left(\left(\frac{\gamma_{T}}{\overline{\gamma_{1}}}\right)^{N_{R}}\right) .
$$

Similarly, we have

$\frac{\Gamma\left(N_{T}, \frac{\gamma_{T}}{\bar{\gamma}_{2}}\right)}{\Gamma\left(N_{T}\right)}=1-\frac{\left(\frac{\gamma_{T}}{\gamma_{1}}\right)^{N_{T}}}{\Gamma\left(N_{T}+1\right)}\left(\frac{1}{\kappa}\right)^{N_{T}}+O\left(\left(\frac{\gamma_{T}}{\overline{\gamma_{2}}}\right)^{N_{T}+1}\right)$.

Substituting (28) and (29) into (19) yields (20), that concludes the proof.

\section{REFERENCES}

[1] M. Duarte, C. Dick, and A. Sabharwal, "Experiment-driven characterization of full-duplex wireless systems," IEEE Trans. Wireless Commun., vol. 11, no. 12, pp. 4296-4307, December 2012.

[2] S. Goyal, P. Liu, S. Panwar, R. Difazio, R. Yang, and E. Bala, "Full duplex cellular systems: will doubling interference prevent doubling capacity?" IEEE Commun. Mag., vol. 53, no. 5, pp. 121-127, May 2015.

[3] D. Kim, H. Lee, and D. Hong, "A survey of in-band full-duplex transmission: From the perspective of PHY and MAC layers," IEEE Commun. Surveys Tuts., vol. 17, no. 4, pp. 2017-2046, 2015.

[4] Y. Liao, L. Song, Z. Han, and Y. Li, "Full duplex cognitive radio: a new design paradigm for enhancing spectrum usage," IEEE Commun. Mag., vol. 53, no. 5, pp. 138-145, May 2015.

[5] G. Liu, F. Yu, H. Ji, V. Leung, and X. Li, "In-band full-duplex relaying: A survey, research issues and challenges," IEEE Commun. Surveys Tuts., vol. 17, no. 2, pp. 500-524, Secondquarter 2015.

[6] A. Sabharwal, P. Schniter, D. Guo, D. Bliss, S. Rangarajan, and R. Wichman, "In-band full-duplex wireless: Challenges and opportunities," IEEE J. Sel. Areas Commun., vol. 32, no. 9, pp. 1637-1652, Sept 2014.

[7] H. Cui, M. Ma, L. Song, and B. Jiao, "Relay selection for two-way full duplex relay networks with amplify-and-forward protocol," IEEE Trans. Wireless Commun., vol. 13, no. 7, pp. 3768-3777, July 2014.

[8] I. Krikidis, H. Suraweera, P. Smith, and C. Yuen, "Full-duplex relay selection for amplify-and-forward cooperative networks," IEEE Trans. Wireless Commun., vol. 11, no. 12, pp. 4381-4393, December 2012.

[9] H. Q. Ngo, H. Suraweera, M. Matthaiou, and E. Larsson, "Multipair full-duplex relaying with massive arrays and linear processing," IEEE J. Sel. Areas Commun., vol. 32, no. 9, pp. 1721-1737, Sept 2014.

[10] T. Riihonen, S. Werner, and R. Wichman, "Mitigation of loopback selfinterference in full-duplex MIMO relays," IEEE Trans. Signal Process., vol. 59, no. 12, pp. 5983-5993, Dec 2011.

[11] H. Suraweera, I. Krikidis, G. Zheng, C. Yuen, and P. Smith, "Lowcomplexity end-to-end performance optimization in MIMO full-duplex relay systems," IEEE Trans. Wireless Commun., vol. 13, no. 2, pp. 913927, February 2014.

[12] K. Yang, H. Cui, L. Song, and Y. Li, "Efficient full-duplex relaying with joint antenna-relay selection and self-interference suppression," IEEE Trans. Wireless Commun., vol. 14, no. 7, pp. 3991-4005, July 2015.

[13] M. Zhou, L. Song, Y. Li, and X. Li, "Simultaneous bidirectional link selection in full duplex MIMO systems," IEEE Trans. Wireless Commun., vol. 14, no. 7, pp. 4052-4062, July 2015. 


$$
\begin{aligned}
F_{\gamma}\left(\gamma_{T}\right)=1-\sum_{i=1}^{\varrho(\boldsymbol{\Sigma})} \sum_{j=1}^{\tau_{i}(\boldsymbol{\Sigma})} \sum_{k=0}^{j-1} \frac{\chi_{i, j}(\boldsymbol{\Sigma})}{k !}\left(\frac{\gamma_{T}}{\overline{\gamma_{1}} \alpha_{\langle i\rangle}}\right)^{k} e^{-\frac{\gamma_{T}}{\bar{\gamma}_{1} \alpha} \sum_{\langle i\rangle}^{k}\left(\begin{array}{c}
k \\
l
\end{array}\right)\left(\gamma_{T}+c\right)^{l} \sum_{m=1}^{\varrho(\boldsymbol{\Lambda})} \sum_{n=1}^{\tau_{m}(\boldsymbol{\Lambda})}} \\
\quad \times \chi_{m, n}(\boldsymbol{\Lambda}) \frac{\left(\overline{\gamma_{2}} \beta\langle m\rangle\right)^{-n}}{\Gamma(n)} e^{-\frac{\gamma_{T}}{\bar{\gamma}_{2} \beta}\langle m\rangle} \sum_{p=0}^{n-1}\left(\begin{array}{c}
n-1 \\
p
\end{array}\right) \gamma_{T}^{n-p-1} \int_{0}^{\infty} w^{p-l} e^{-\frac{\gamma_{T}^{2}+\gamma_{T}}{w \overline{\gamma_{1}} \alpha\langle i\rangle}} e^{-\frac{w}{\bar{\gamma}_{2} \beta}\langle m\rangle} d w .
\end{aligned}
$$

[14] Z. Zhang, X. Chai, K. Long, A. Vasilakos, and L. Hanzo, "Full duplex techniques for 5G networks: self-interference cancellation, protocol design, and relay selection," IEEE Commun. Mag., vol. 53, no. 5, pp. 128-137, May 2015.

[15] G. Amarasuriya, C. Tellambura, and M. Ardakani, "Performance analysis of hop-by-hop beamforming for dual-hop MIMO AF relay networks," IEEE Trans. Commun., vol. 60, no. 7, pp. 1823-1837, July 2012.

[16] N. Ferdinand and N. Rajatheva, "Unified performance analysis of twohop amplify-and-forward relay systems with antenna correlation," IEEE Trans. Wireless Commun., vol. 10, no. 9, pp. 3002-3011, September 2011.

[17] R. H. Louie, Y. Li, H. Suraweera, and B. Vucetic, "Performance analysis of beamforming in two hop amplify and forward relay networks with antenna correlation," IEEE Trans. Wireless Commun., vol. 8, no. 6, pp. 3132-3141, June 2009.

[18] G. Zhu, C. Zhong, H. Suraweera, Z. Zhang, and C. Yuen, "Outage probability of dual-hop multiple antenna af systems with linear processing in the presence of co-channel interference," IEEE Trans. Wireless Commun., vol. 13, no. 4, pp. 2308-2321, 2014.

[19] X. Xia, K. Xu, D. Zhang, and Y. Xu, "Low-complexity transceiver design and antenna subset selection for cooperative half- and full-duplex relaying systems," in Proc. IEEE GLOBECOM '14, Dec 2014, pp. 33143319.

[20] T. Riihonen, S. Werner, and R. Wichman, "Hybrid full-duplex/halfduplex relaying with transmit power adaptation," IEEE Trans. Wireless Commun., vol. 10, no. 9, pp. 3074-3085, September 2011.

[21] X. Xia, W. Xie, D. Zhang, K. Xu, and Y. Xu, "Multi-pair full-duplex amplify-and-forward relaying with very large antenna arrays," in Proc. IEEE WCNC '15, March 2015, pp. 304-309.

[22] $\mathrm{H}$. Ju, E. Oh, and D. Hong, "Improving efficiency of resource usage in two-hop full duplex relay systems based on resource sharing and interference cancellation," IEEE Trans. Wireless Commun., vol. 8, no. 8, pp. 3933-3938, August 2009.

[23] Y. Y. Kang and J. H. Cho, "Capacity of MIMO wireless channel with full-duplex amplify-and-forward relay,' in Proc. IEEE PIMRC '09, Sept 2009, pp. 117-121.

[24] Z. Ding, K. Leung, D. Goeckel, and D. Towsley, "On the application of cooperative transmission to secrecy communications," IEEE J. Sel. Areas Commun., vol. 30, no. 2, pp. 359-368, February 2012.

[25] A. Almradi and K. Hamdi, "Ergodic capacity analysis of MIMO full duplex relaying with imperfect CSI," in Proc. IEEE GLOBECOM, Dec 2015, pp. 1-6.

[26] — , "Outage probability of spatially correlated MIMO full-duplex relaying with imperfect CSI," in Proc. IEEE WCNC'16, Apr 2016, pp. 1328-1333.

[27] H. Shin and M. Win, "Mimo diversity in the presence of double scattering," IEEE Trans. Inf. Theory, vol. 54, no. 7, pp. 2976-2996, July 2008.

[28] M. Hasna and M.-S. Alouini, "End-to-end performance of transmission systems with relays over Rayleigh-fading channels," IEEE Trans. Wireless Commun., vol. 2, no. 6, pp. 1126-1131, Nov 2003.

[29] S. Ikki and S. Aissa, "Performance analysis of two-way amplify-andforward relaying in the presence of co-channel interferences," IEEE Trans. Commun., vol. 60, no. 4, pp. 933-939, April 2012.

[30] H. Bolcskei, M. Borgmann, and A. Paulraj, "Impact of the propagation environment on the performance of space-frequency coded MIMOOFDM," IEEE J. Sel. Areas Commun., vol. 21, no. 3, pp. 427-439, Apr 2003.

[31] I. S. Gradshteyn and I. M. Ryzhik, Tables of Integrals, Series and Products. 6th ed. San Diego, CA, USA: Academic, 2000.

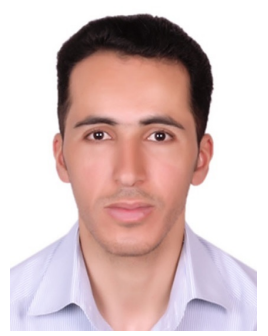

Ahmed Almradi (S'14) received the B.Sc. degree in Electrical and Electronic Engineering in 2004 from the University of Tripoli, Tripoli, Libya, then received the M.Sc. degree in Electrical Engineering in 2012 from Rochester Institute of Technology, Rochester, NY, USA. He is on study leave from Azzaytuna University, Tarhuna, Libya. He is currently working towards the Ph.D degree at the University of Manchester, Manchester, U.K. His research interests are in the modeling, design, and performance analysis of wireless communication systems with special emphasis on MIMO half-duplex and full-duplex relaying systems, wireless information and power transfer (energy harvesting) systems, diversity and beamforming, and OFDM systems.

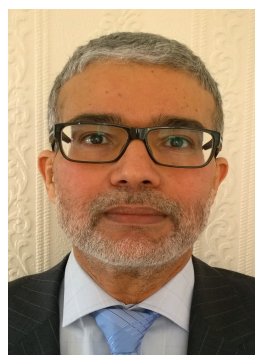

Khairi Ashour Hamdi (M'99-SM'02) received the B.Sc. degree in Electrical Engineering from the University of Tripoli, Tripoli, Libya, in 1981; the M.Sc degree (with distinction) from the Technical University of Budapest, Budapest, Hungary, in 1998; and the Ph.D. degree in Telecommunication Engineering from Hungarian Academy of Sciences, Budapest, in 1993. He was with the University of Essex, Colchester, U.K. He is currently with the School of Electrical and Electronic Engineering, The University of Manchester, Manchester, U.K. His current research interests include modelling and performance analysis of wireless communication systems and networks, green communication systems, and heterogeneous mobile networks. 